Institut Curie, PSL Research University, CNRS UMR3348, Centre Universitaire, Bâtiment 110, F-91405 Orsay, France. carsten.janke@curie.fr

\section{Andrea Musacchio}

Department of Mechanistic Cell Biology, Max Planck Institute of Molecular Physiology, Otto-HahnStrasse 11, 44227 Dortmund, Germany; and the Centre for Medical Biotechnology, Faculty of Biology, University Duisburg-Essen, Universitätsstrasse, 45141 Essen, Germany. andrea.musacchio@mpi-dortmund.mpg.de

\section{Eva Nogales}

Biochemistry, Biophysics and Structural Biology Division, Molecular and Cell Biology Department, University of California, Berkeley, California 94720-3220, USA; the Howard Hughes Medical Institute, Maryland, USA; and the Lawrence Berkeley National Laboratory, USA. enogales@lbl.gov

\section{Abstract}

Next year will be the 50th anniversary of the discovery of tubulin. To celebrate this discovery, six leaders in the field of microtubule research reflect on key findings and technological breakthroughs over the past five decades, discuss implications for therapeutic applications and provide their thoughts on what questions need to be addressed in the near future.

Identifying the main component of microtubules was obviously the pressing task in the field around 50 years ago. What key questions were being pursued when you entered the arena of microtubule research?

Gary Borisy. Actually, I had no idea in the early 1960s that I was entering the arena of microtubule research, because it hadn't yet been defined as an arena. Although the term 'microtubule' was first coined in 1963, the structures had not, in fact, been observed in most cells, because the fixation methods used at the time did not preserve them. It was only after the introduction of glutaraldehyde as a fixative, also in 1963, that they began to be observed routinely. The concept of the microtubule as a 'ubiquitous' cytoskeletal structure wasn't put forward until 1965. My entry point into the field was mitosis: I wanted to get a molecular handle on how cells divide. Working with Ed Taylor at the University of Chicago (Illinois, USA), we were trying to identify and purify the molecule that bound to colchicine, because colchicine was known to specifically inhibit mitosis. We had no preconceived idea about what the colchicine target would be, but we believed that identifying the target would teach us something important about mitosis. So, the key questions for us at the time were how to isolate, purify and characterize the colchicine-binding protein and then to establish its identity ${ }^{1,2}$.

Rebecca Heald. In the mid-1990s, one pressing question was why microtubules in cells were so much more dynamic than microtubules assembled from purified tubulin. Neuronal microtubule-associated proteins (MAPs) had been identified and studied (in large part because they co-purified with tubulin isolated from brain tissue, where it is most abundant), but these proteins all stabilized microtubules, and factors that induced the transition from growth to shrinkage (catastrophe) were unknown. A related question was how the 
microtubule cytoskeleton transformed from a relatively stable interphase array to a highly dynamic bipolar spindle in mitosis. At the time, the centrosome was thought to be the sole 'microtubule-organizing' centre of the cell, determining the site of microtubule growth and their polarized orientation. In my opinion, the discovery of a large family of kinesin motor proteins, as well as cytoplasmic dynein, spawned key investigations into how cellular factors affect microtubule behaviour. The diverse activities of different motor proteins to induce catastrophe, crosslink and move microtubules relative to one another revealed the ability of microtubule arrays to 'self-organize'. This process allows the spindle to form in the absence of centrosomes - for example, during female meiosis in many animal species, or when the centrosome is inactivated genetically or by laser ablation. An important ongoing challenge is to fully understand how microtubule dynamics and organization emerge from a defined set of proteins through reconstitution experiments.

Jonathon Howard. One of the big questions back when I got into the microtubule business, around 1990, was how motor proteins such as kinesin and dynein use ATP hydrolysis to generate force for transport along microtubules (such as axonal transport) or for cell motility (such as ciliary or flagellar motion). The interaction of kinesin with microtubules was a model system, because it was clear that only a relatively small number of kinesins must be capable of moving small vesicles along microtubules. A related question was how microtubule growth and shrinkage could generate force to move chromosomes during mitosis. Polymerization and depolymerization forces were very mysterious: how could you hold on to the end of a depolymerizing microtubule? How could a microtubule grow, and new tubulin subunits get in, if its end was pushing up against something? What role did the GTP cap have, and how was energy from GTP used to generate pulling or pushing forces? How did MAPs regulate growth and shrinkage?

Carsten Janke. I entered the field of microtubule research somewhat through a back door. During my Ph.D. studies, I worked on the role of the MAP tau in neurodegeneration, and in my postdoctoral work, I characterized new kinetochore protein complexes in budding yeast. This was in the late 1990s, and at the time, the field had already expanded a lot: different research communities pursued their own interests. There were many parallel advances at the time, such as the biochemical and functional dissection of the kinetochore, the understanding of the role of primary cilia as the 'cell antennae', and advances in the characterization of neuronal transport mediated by microtubules. Specifically regarding microtubule research, I think a highlight of the 1990s and 2000s was the use of highly sophisticated, in vitro reconstructions of microtubule assemblies from recombinant components, and their biophysical characterization. This allowed the definition of minimal functional units of microtubule assemblies such as the microtubule arrays of the mitotic spindle. A second highlight was the amazing advances in imaging of microtubule structures in vivo, which were driven by substantial improvements in light microscopy.

Andrea Musacchio. When I entered the field, approximately 15 years ago, studies on my main research interest, the kinetochore, had remained traditionally distinct from studies of microtubules as cytoskeletal components. The search-and-capture model offered a conceptual basis for how microtubules that interact with kinetochores might become selectively stabilized to generate kinetochore fibres, but the mechanism was obscure. The 
critical gap in knowledge was in our understanding of how kinetochores, which are among the largest (if not the largest) microtubule-binding machines, bind to and stabilize microtubules to link them to chromosomes. Research therefore focused on the identification of potential linking proteins and how they are harnessed to align the sister chromatids to the metaphase plate, and later to part them to the opposite spindle poles. The identification of an array of new kinetochore proteins in the early 2000s facilitated the identification of such proteins. Most notably, teams led by Arshad Desai (University of California San Diego, USA) and Ted Salmon (University of North Carolina, Chapel Hill, USA), with co-workers that included Iain Cheeseman (now at the Whitehead Institute in Cambridge, Massachusetts, USA) and Jennifer DeLuca (now at Colorado State University in Fort Collins, USA), identified in 2006 the NDC80 complex as a crucial component of this physical linkage. Our laboratory has been particularly interested in understanding the structural organization of these kinetochore components and their interactions with microtubules.

Eva Nogales. Among my more vivid scientific memories when I was a graduate student working on tubulin self-assembly at Daresbury Laboratory, Cheshire, UK, are the reports by Eva-Maria and Eckhard Mandelkow (then at the Max Planck Unit for Structural Biology, Hamburg, Germany) and Marie-France Carlier (at the National Centre for Scientific Research (CNRS), Paris, France) of really fascinating microtubule 'oscillations', in which a whole population of microtubules was synchronized to undergo periods of assembly and disassembly in the test tube. It was also very interesting that a number of antimitotic agents that targeted tubulin and affected dynamic instability were being discovered.

But what I remember as the most exciting development at the time was the publication of the structure of actin by Ken Holmes and colleagues (Max Planck Institute at Heidelberg, Germany). It became obvious that there was also a pressing need for a structure of tubulin. Many were trying to crystallize it, like actin, in an inhibited form. At the same time, the new technique of cryoelectron microscopy (cryo-EM) started to be used on cytoskeletal filaments. This was the time when Dick Wade (CNRS) was imaging microtubules and proposed the lattice accommodation theory to explain the existence of microtubules with different protofilament numbers. Also using cryo-EM, the Mandelkows and Ron Milligan (Scripps Research Institute, California, USA) visualized the depolymerization of microtubules by protofilament peeling, producing images that inspired me and influenced my scientific career.

Which technical breakthrough or breakthroughs, in your opinion, have been the most significant in driving microtubule research? What questions did particular techniques enable scientists to address?

G.B. One breakthrough was demonstrating that colchicine did, in fact, bind to a specific molecule. In retrospect, colchicine was one of the first examples of how a small-molecule probe could be used to explore a biological pathway. However, the task of identifying the colchicine target with the subunit of microtubules was not straightforward, as the biochemistry was confusing and messy. A breakthrough was in finding a good source of colchicine-binding activity from which the protein could be isolated. This, surprisingly, turned out to be brain tissue - which we now understand to be logical, because 
microtubules are a principal structural component of neurons. Another breakthrough was the discovery of how to reassemble microtubules in vitro from the purified protein. This permitted analysis of the mechanisms of assembly and disassembly, and the identification of proteins that specifically interacted with microtubules. A decade before the advent of GFP, the use of fluorescently labelled tubulin micro-injected into cells led to the realization that microtubules were highly dynamic structures, transforming our concepts of the cytoskeleton. The concept of dynamic instability gave new insights into how cytoplasmic microtubules could be rapidly remodelled. The discovery of molecular motors and that microtubules serve as tracks for transport of cellular cargo transformed our understanding of molecular traffic in the cell.

R.H. Innovations in microscopy, particularly fluorescence and electron microscopy, have been instrumental in revealing the basis of microtubule dynamics and organization. Timelapse video microscopy was required to directly visualize the growth and shrinkage of individual microtubules within a population, as well as the stochastic transition between these two states, and enabled precise measurement of these dynamic parameters. This was a prerequisite for investigating how specific proteins affected microtubule behaviour, for example, by altering growth rate or the frequency of catastrophes. The development of fluorescently tagged tubulin was of great significance, and techniques such as fluorescent speckle microscopy and other approaches that labelled one end of a microtubule or generated traceable fiduciary marks along its length permitted tracking of microtubule movements inside cells. This enabled researchers to fully characterize dynamic changes in microtubule organization, for example, in migrating cells or dividing cells. To elucidate the structural basis of microtubule dynamic instability, it was essential to obtain high-resolution images, and the development of cryo-EM and image-analysis tools have been instrumental. These tools are being applied to gain detailed insight into the features of tubulin that underlie the amazing intrinsic behaviour of microtubules, as well as important information about how specific proteins associate with microtubules, and may even reveal what lurks inside!

J.H. Single-molecule techniques had a huge impact. Detecting single molecules, especially using total internal reflection fluorescence microscopy (TIRF), but also manipulating them with optical traps, really blew open the problem of how proteins generate force. The experimental obstacle back then was how to assay the movement of a motor protein, such as kinesin, along its filament, the microtubule. In the case of DNA, the position of a RNA polymerase, which can be thought of as another type of motor protein, could be determined biochemically by footprinting - that is, sequencing the short but unique oligonucleotide that the enzyme protects from proteolysis. This even had single-nucleotide resolution! In the case of cytoskeletal filaments such as microtubules or actin filaments, this approach did not work, because the filaments are periodic as all the subunits are the same. Single-molecule techniques allowed the movement of individual motors to be directly visualized. It was discovered that many cytoskeletal motor proteins, such as kinesin-1 (REF. 3), were processive, meaning that they could take many steps along their filament, like RNA and DNA polymerases. This facilitated the detailed study of their mechanisms (using purified proteins) or their functions (using labelled proteins in living cells). As optics techniques, 
labelling strategies, detectors and image processing improved, it became possible to track proteins with nanometre precision ${ }^{4}$, enabling the progression of motors and the growth of microtubules to be studied at the single-molecule and single-subunit level. The fluorescence techniques, in all of their different 'flavours', could even be used inside living cells, allowing biochemical studies - of binding kinetics, oligomerization, activity state and so on - to be done in situ.

C.J. In the early years of microtubule research, major advances in electron microscopy and biochemistry drove the discovery of the microtubule, then the discovery of tubulin, and, shortly after, the discovery of the detyrosination-tyrosination cycle of a-tubulin - which was an amazing finding, as it showed that amino acids can be enzymatically incorporated into proteins independently of an RNA template and translation. Advances in cloning techniques led to the discovery, at the end of the 1970s, of multiple $\alpha$ - and $\beta$-tubulin genes. Later, the development of recombinant protein production, together with biochemistry using Xenopus laevis egg extracts, enabled great progress in the field, as they allowed us to form microtubule spindles in these extracts and study the role of specific proteins by removing them from or adding them into the extracts. The huge success of monoclonal and (especially) peptide antibodies was also one of the driving forces of microtubule research, as these allowed detection of different tubulin subtypes, such as tubulin isotypes and posttranslational modifications, in the 1980s. Later, as I mentioned above, improvements in light microscopy and biochemical reconstructions of microtubule assemblies in vitro were the key technologies that advanced the field. Interestingly, when looking back, it seems that not only have technological advances helped the microtubule field, but research on microtubules has also driven many of these technological advances. We certainly hope that this synergy will continue in the future.

A.M. The data obtained by in vitro reconstitution of interactions between MAPs and microtubule motors with static or dynamic microtubules using fluorescence microscopy have superseded the data obtained by the murky, poorly characterized assays that had previously been used for measuring microtubule binding, nucleation or bundling. The use of fluorescence microscopy enabled quantitative analyses of microtubule dynamics. Such experiments can now be complemented by technically more demanding force measurements with optical or magnetic tweezers. Real-time measurements, often achieving singlemolecule sensitivity, are revealing the variety of interaction modes that MAPs and motors establish with microtubules, the dynamics of their residency on microtubules, and the consequences of their binding on microtubule stability and growth rates. Studies on the MAPs XMAP215 and end-binding protein 1 (EB1) come to mind in this context, but there are many other noteworthy examples. Structural biology has also had a primary role in microtubule research. For instance, the recent elucidation of the structures of dynein and dynactin, and of tubulin and microtubules, at atomic or near-atomic resolution are clear landmarks. The revolutionary recent developments in cryo-EM hold great promise for future elucidations of the mechanistic basis of microtubule function.

E.N. I am biased towards visualization tools! Ted Salmon and Clare Waterman (University of North Carolina) pioneering speckle microscopy has enabled many to visualize cellular microtubules, whether they are moving, growing, shrinking or treadmilling. But, of course, 
cryo-EM remains among my personal favourites. A number of laboratories, including those of Milligan, Wade and Linda Amos (MRC Laboratory of Molecular Biology, Cambridge, UK), produced the first cryo-EM structures of microtubules decorated with kinesin, in which the crystal structure of kinesin, then just solved by Ron Vale (University of California San Francisco), could be docked into the density maps. And then the structure of tubulin itself was obtained using electron crystallography of zinc-induced sheets through the work of Sharon Wolf and myself, under the leadership of Ken Downing (Lawrence Berkeley National Laboratory, California). Cryo-EM continues to produce beautiful molecular images of complex cellular microtubule structures. Among my favourites are the structures by Daniela Nicastro (Brandeis University and UT Southwestern, USA) of whole axonemes. Our most detailed visualization of tubulin has come from X-ray structures, pioneered by Marcel Knossow (CNRS), and more recently describing the binding of several antimitotic agents by Michel Steinmetz (Paul Scherrer Institut, Switzerland). Finally, I love what the field of single-molecule biophysics is doing to increase our mechanistic understanding of microtubule motors, using single-molecule fluorescence, Förster resonance energy transfer (FRET) or laser tweezers, with contributions from Ron Vale, Steve Block (Stanford University, California), Ahmet Yildiz (University of California Berkeley) or Sam ReckPeterson (Harvard University, Cambridge, Massachusetts), to cite a few.

How successfully has our accumulated knowledge of microtubule structure and function been translated to the clinic, in terms of our understanding of how microtubules drive disease and their importance as possible therapeutic targets? What facets are limiting translation and how might they be addressed?

G.B. So many extraordinary technological advances over the past several decades have revolutionized biology in general and of course had a profound impact on our understanding of microtubule structure and function. DNA cloning and sequencing enabled the identification of the tubulin gene family and the enormous variety of microtubule-interacting proteins. X-ray crystallography and cryo-EM have provided structures of tubulin and microtubules at the atomic level. Genetically encoded fluorescent proteins such as GFP have enabled analysis of dynamic functions in vivo. The revolution in imaging has enabled unprecedented spatial and temporal visualization of cellular components. One striking example of translating this knowledge to the clinic is the use of taxol as an anticancer drug. Taxol binds to microtubules and stabilizes them by inhibiting their disassembly, thus freezing their dynamics. Taxol has proven to be a powerful therapeutic for breast and ovarian cancer. However, much greater opportunities for translational impact remain. Microtubules are involved in biological processes in virtually every cell, tissue and organ in the body, and microtubule disturbances underlie many diseases such as Alzheimer disease and Parkinson disease, as well as cancer. So, the potential of microtubule-based therapeutics is great. However, the microtubule system is very complex, and achieving full translational impact will require a deconstruction of that complexity — what today would be called a systems biology approach.

R.H. Microtubule-targeting drugs are of great value in the clinic, but it seems that we do not fully understand how and why they work. For cancer therapies, microtubule-targeting drugs affect the spindle and cell division. In my opinion as a cell biologist, translation is limited by 
our incomplete knowledge of the spindle, and basic research into how the spindle forms and functions will be instrumental in informing therapeutic approaches. This requires the contributions of researchers working in a wide variety of systems and organisms, not just cultured cell-based assays, together with an open-minded and curious attitude towards the underlying biology. Increasing the emphasis on 'translational' research is not going to solve the problem of finding effective therapeutic approaches if we do not understand how the microtubule cytoskeleton operates in the context of a cell, tissue, and organism.

J.H. Given the central role that microtubules have in cell division, in cilia and flagella, and in intracellular transport, especially in neurons, it is not surprising that many genetic diseases have been traced to defects in microtubule motors and other associated proteins. Taxol, the microtubule-stabilizing diterpenoid isolated from the bark of yew (used in the witches brew), was an essential reagent for studying microtubules in vitro. Before a synthesis was developed, which was an incredible feat, taxol was only available in miniscule quantities from the US National Institutes of Health (NIH). Taxol has been very successful in treating ovarian and other cancers. But magic bullets like taxol are rare; I think systems approaches to therapy in which multiple, softer tools push the organism back into a healthy, hopefully stable state offer a promising avenue in the future.

C.J. Microtubules are a prime target of cancer chemotherapy: taxol derivatives and vinca alkaloids are microtubule poisons that account for one-third of all chemotherapeutic drugs used. While it is extremely successful, this type of treatment is very crude, as it affects the entire organism and perturbs many cellular functions, and thus it has considerable side effects. Furthermore, some types of cancers develop resistance to this treatment, notably by expressing a specific tubulin gene, TUBB3, which encodes the otherwise neuron-specific tubulin $\beta 3$ isotype. Microtubules containing this isotype appear less susceptible to taxol. Considerable efforts are underway to find new microtubule drugs that could act more specifically on cancer cells, such as antibody-drug conjugates that deliver tubulin drugs specifically into cancer cells. Another therapeutic tactic could be to develop drugs that are specific to particular forms of tubulin, such as isotypes or post-translationally modified tubulins; however, so far no cancer-specific tubulin forms have been identified. Of course, there are also drugs that affect other essential microtubule-interacting proteins that are being actively pursued.

Considering the importance of microtubules in other clinical conditions, tubulin drugs hold great promise for the treatment of ciliopathies and neurodegenerative disorders, as at least a subset of these pathologies are linked to failures in microtubule function. Finally, some very exciting reports were published recently on the positive effects of taxol and another tubulin drug, epothilone B, on axon regeneration. Overall, I think there is a greatly underestimated potential in tubulin drugs.

A.M. I will limit myself to a few general comments. There is clearly a rationale in targeting microtubules in disease, with cancer being a primary example, but the molecular basis for microtubule targeting remains incompletely understood. At the end of the last decade, livecell microscopy began to be exploited to study how mitotic cells behave when treated with agents that interfere with microtubule function (microtubule poisons) or with the function of 
the mitotic checkpoint, which becomes activated in the presence of such poisons. In this context, a crucial question was whether some aspects of tumour cells - for instance, preexisting aneuploidy - might explain the differential sensitivity of tumour cells to microtubule poisons and therefore be exploited for therapy. The results that have been obtained so far are complex and have not yet resolved the issue in full. Nevertheless, stratification and selection of potential drug targets based on defined cellular behaviours continues to be an appealing therapeutic approach. Eventually, selected combinations of drugs might be harnessed to force cancer cells into particular cell cycle stages - most notably, mitosis - in which a crucial dependence (and thus weakness) can be exposed.

E.N. I think that there is still a long way to go. We do not yet fully understand microtubule function and dynamics in healthy cells and organisms, so our understanding of their role in disease states remains limited. This lack of a comprehensive understanding stems from the complexity of the microtubule cytoskeleton and the many roles that microtubules have in the cell. The functional complexity is paralleled by the molecular complexity of tubulin isoforms, their post-translational modifications and the myriad of cellular factors that regulate tubulin transcription and translation, folding of the $\alpha \beta$-dimer, and microtubule nucleation and assembly, as well as microtubule localization and organization. It is clear that many questions remain to be answered, and the number of questions may continue to increase the more we learn about these fascinating polymers. My work is very distant from applications in the clinic, but we know that the effects of some broadly used anti-tubulin drugs for the treatment of cancer are still not well defined. For example, it is not entirely understood how the administration of taxol ultimately results in tumour remission, as it is most likely that taxol does more than just causing mitotic arrest. More basic as well as translational and clinical work is clearly necessary to develop improved therapies.

As the field of microtubule research has matured in the five decades since tubulin was identified as the main component of these cellular structures, which of the emerging basic principles have you found most unexpected? What question are you most intrigued about now?

G.B. Of particular interest to me are the qualities of cellular organization that go beyond anything yet engineered by us humans. Cells are ensembles of molecules interacting within boundaries. Some of the molecules are organized into supramolecular assemblies that have been likened to molecular machines. But the machine metaphor is rather limiting if we imagine machines of the human-made type such as we see around us. What human-made machine assembles itself from parts? What machine is constantly in material flux, tossing out parts and taking in new ones? What machine can repair itself? Yet these are all properties of many of the molecular machines found in cells — including the microtubule machine. Organization in the cell depends on a continuity of form, not on individual molecules. It operates far from equilibrium and disintegrates if not supplied with an input of energy. A big challenge for cell biology is to understand the emergent, self-organizing properties of interdependent molecular systems. It is likely that a successful response to this challenge will require multidisciplinary approaches, including a heavy dose of biophysics, systems analysis and computer modelling. It may be, following a quote from Richard Feynman, that 
we will not be able to fully understand these systems until we can create them. The frontier may well be synthetic cell biology.

R.H. The most amazing aspect of the microtubule cytoskeleton to me is that the basic components are conserved across species, yet a tremendous diversity of microtubule-based structures can form in different cell types and organisms. Compare microtubule arrays in a tiny $2 \mu \mathrm{m}$ yeast cell to those in a giant $2 \mathrm{~mm}$ single-cell protist like Stentor! Microtubules have essentially the same structure and similar associated factors, and carry out some common and some very different functions, in these two cell types. How are vastly different microtubule arrays assembled and remodelled using the same basic cytoskeletal system? My laboratory is obsessed with understanding the mitotic spindle, whose architecture and size adapt to fit a wide variety of cell types. Can we leverage evolutionary differences to elucidate mechanisms that modulate microtubule dynamics and organization, thereby setting spindle size and shape? How do these mechanisms impact the fidelity of chromosome segregation by the spindle? Cancer cells are almost always distorted in size and morphology, and expression of spindle-associated proteins is frequently altered. Investigating the molecular basis of spindle variation across species and cell types is likely to reveal new principles of microtubule regulation and may identify important factors that contribute to genome instability and oncogenesis.

J.H. The discovery I found most unexpected was that the kinesin-related protein NCD (of the kinesin-14 family) moves backwards! That is, it moves in the opposite direction to kinesin-1, the founding member of the family. In addition to posing all sorts of questions about the structural basis of motor directionality, this discovery presaged the extraordinary range of activities of kinesins: some move one way, some the other way, and some can move both ways; some can slide microtubules, while some depolymerize microtubules and some can even polymerize them! In addition, there are many other proteins, such as XMAP215 and EB1, that also regulate the dynamic growth and shrinkage of microtubules and that couple microtubule ends to a large variety of cellular structures - for example, chromosomes, basal bodies, centrosomes and endoplasmic reticulum — and figuring out how all this works is a tremendous challenge.

The question that most intrigues me now is the length control problem: what sets the size of microtubules and microtubule-based structures. How do small molecules like tubulin, motors and MAPs know how long a microtubule is? This is a mechanical signalling question, and we have ideas about how motor activity can feed back on microtubule dynamics to control length.

C.J. I'm not certain about some of the early discoveries, but the discovery by Tim Mitchison and Marc Kirschner (at the time at the University of California San Francisco) of the dynamic instability of microtubules was certainly a great breakthrough. I also think that the discovery of enzymatic detyrosination and tyrosination was a great surprise at the time, as it showed that gene-encoded amino acid sequences can be modified by enzymes. More recently, I found the discovery of microtubule self-repair very surprising and exciting, since it had always been thought that microtubules only change their composition by depolymerization and repolymerization, not by insertion of tubulin units into the lattice. This 
discovery reopened the great question of how perfectly microtubules are assembled in our cells.

I am personally driven by my interest in the tubulin code, which turns out to be a very challenging code to crack. As we know today, the entire diversity of the cellular tubulin pool, which is generated by the expression of different tubulin genes (isotypes) and by tubulin post-translational modifications, leads in most cases to only subtle changes in microtubule behaviour, changes that are difficult to measure with classical methods. I guess this could be another example in which technological advances will be needed to appreciate the full scale of microtubule functions that are regulated by the tubulin code.

A.M. Structural biologists like myself are usually addressing systems whose parts selfassemble in conditions of thermodynamic equilibrium. Microtubule assembly ultimately defies this approach because it is dissipative, and this allows more flexible biochemical behaviours. Using some tricks, microtubules are of course amenable to structural analysis, but their nature is to behave dynamically. The description of the dynamic instability of microtubules ${ }^{5}$ and the development of a theory for how it may influence cellular morphogenesis - the search-and-capture model ${ }^{6}$ of Tim Mitchison and Marc Kirschner (both at the Harvard Medical School) - has revolutionized the field. The fundamental concept behind this model is that self-organization emerges from the dynamic behaviour of the constituent parts, which in turn reflects their ability to operate away from thermodynamic equilibrium. I suspect that future inspiration at this point is likely to arrive from the reconstitution of microtubule-based macromolecular structures of adequate complexity, where the simultaneous control of multiple parameters is possible - something that is still very difficult to do in non-reconstituted systems. To be able to gain a holistic view of self-organization of microtubule-based structures, we must increase the complexity of the reconstituted systems well beyond current limits. We try to keep these concepts in mind as we reconstitute larger and larger parts of the kinetochore to understand its dynamic interactions with microtubules. I wish microtubules a good anniversary!

E.N. How antimitotic agents interfere with microtubule dynamic instability is something I have been interested in since my Ph.D., and it remains close to my heart. But I am also fascinated by how cellular factors regulate dynamic instability, and how others factors utilize microtubule dynamics for their own purposes, such as moving with a growing or shrinking microtubule end. There has been some beautiful imaging of these molecular interactions, as well as single-molecule force studies, which have provided unique mechanistic insights such as the work by Thomas Surrey (at the Francis Crick Institute, London, UK) on endbinding proteins, by Joe Howard (Yale University, New Haven, Connecticut, USA) on the microtubule polymerase XMAP215, or by Stefan Westermann (Institute of Molecular Pathology, Vienna, Austria) and Chip Asbury (University of Washington, USA) on kinetochores, to cite some of my own favourites - that have been real eye-openers for me. The complexity of these molecular activities intrigues me and motivates me to study the interaction of microtubules with these regulatory factors at the structural level. Although we have made some progress, both on the understanding of the basic structural principles of microtubule dynamic instability and on the visualization of pairwise interactions between microtubules and associated factors, it is time to look at larger and more complex networks 
of interactions as they occur on the microtubule surface. A combination of biochemical reconstitution approaches and cellular imaging will certainly be needed.

\section{Glossary}

Axonemes

Microtubule-based superstructures that run along the lengths of cilia and flagella. They typically contains two central microtubules surrounded by nine microtubule doublets.

\section{Density maps}

Distribution of mass corresponding to a macromolecule obtained by structural biology methods such as X-ray crystallography or cryoelectron microscopy.

\section{Detyrosination-tyrosination cycle}

A post-translational modification of a-tubulin, consisting of the enzymatic removal of the gene-encoded, carboxy-terminal Tyr residue (by enzymes yet unknown) and its enzymatic re-ligation by tubulin Tyr ligase.

\section{Dynamic instability}

A property of microtubules discovered in 1984 by Tim Mitchison and Mark Kirschner (then at the University of California San Francisco, USA), by which individual microtubules switch stochastically between phases of growth and shrinking, powered by GTP hydrolysis that closely follows incorporation of a tubulin dimer into a growing microtubule end.

\section{Dynein and dynactin}

Dynein is a complex, multi-subunit microtubule minus-end-directed motor. Studies in vitro suggest that its processive movement on microtubules may require dynactin, which is a very large multi-subunit assembly, as well as additional adaptor subunits.

\section{GTP cap}

A GTP-tubulin-rich structure at the growing tip of a microtubule, created by the binding of GTP-bound tubulin subunits from solution onto the microtubule end.

\section{End-binding proteins}

Proteins that bind with higher affinity to a region at the dynamic, growing end of a microtubule.

\section{Fluorescent speckle microscopy}

A live-imaging technique in which a low number of randomly incorporated labelled selfassembling subunits generate fluorescence intensity patterns that appear as distinct puncta, called speckles, that serve as fiduciary markers so that motion and turnover of the polymer can be visualized.

\section{Kinesin}

A family of small ATPases, distantly related to myosins and G proteins, that bind specifically to microtubules and undergo conformational changes upon ATP binding, hydrolysis and product (ADP and inorganic phosphate) release. The conformational changes 
can be used to drive directed motion, to generate force and to alter the growth or depolymerization of microtubules.

\section{Kinetochore}

A large protein assembly of nearly 100 proteins that links centromeric DNA to spindle microtubules, thereby coupling forces generated by microtubule dynamics to power chromosome movement during mitosis and meiosis.

\section{Lattice accommodation theory}

A geometrical model that explains how tubulin contacts can be maintained in microtubules with different protofilaments by skewing of the protofilaments with respect to the microtubule axis.

\section{Optical or magnetic tweezers}

Optical tweezers, also known as optical traps, are instruments that use a focused laser beam to generate forces on micrometre-scale objects. Magnetic tweezers are instruments that can generate forces or torque. Both instruments can operate in biologically meaningful force ranges, typically piconewtons, and are therefore ideally suited to study the interactions of microtubules with motors and microtubule-associated proteins.

\section{Peptide antibodies}

Antibodies raised against synthetic short peptides representing an epitope of a protein. If they are generated for a peptide mimicking a specific post-translational modification, the antibodies can be used to determine the state of this modification at the native protein.

\section{Search-and-capture model}

Proposed by Mitchison and Kirschner in 1886, this model builds on an intrinsic property of microtubules named dynamic instability. The search-and-capture model proposed that this property allows the dynamic ends of microtubules to explore space randomly, until their capture by defined binding targets (for example, kinetochores) endowed with the ability to suppress instability, which would therefore selectively stabilize on-target microtubules. Many aspects of this model have since been confirmed, including the strong stabilization of microtubules after kinetochore binding.

\section{Total internal reflection fluorescence microscopy}

(TIRF). An optical technique that exploits the 'trapping' of light within high-refractive-index structures such as optical fibres and even 'fluorescent' signs such as those sometimes used to advertise menus in cafes or restaurants, in which writing on the glass surface allows the light to leak out.

\section{Tubulin code}

Describes the process of generating specialized microtubules by directly changing the tubulin building blocks, either by the incorporation of specific tubulin isotypes (a set of gene products of $\alpha$ - and $\beta$-tubulins) into the lattice, or by the addition of specific posttranslational modifications to tubulin.

\section{Vinca alkaloids}


A set of natural or semi-synthetic alkaloid agents derived from vinca plants, which have the capacity to inhibit microtubule assembly and are therefore used in cancer therapy.

\section{References}

1. Borisy GG, Taylor EW. The mechanism of action of colchicine. Binding of colchincine- ${ }^{3} \mathrm{H}$ to cellular protein. J. Cell Biol. 1967; 34:525-533. [PubMed: 6068183]

2. Borisy GG, Taylor EW. The mechanism of action of colchicine. Colchicine binding to sea urchin eggs and the mitotic apparatus. J. Cell Biol. 1967; 34:535-548. [PubMed: 6035643]

3. Howard J, Hudspeth AJ, Vale RD. Movement of microtubules by single kinesin molecules. Nature. 1989; 342:154-158. [PubMed: 2530455]

4. Svoboda K, Schmidt CF, Schnapp BJ, Block SM. Direct observation of kinesin stepping by optical trapping interferometry. Nature. 1993; 365:721-727. [PubMed: 8413650]

5. Mitchison T, Kirschner M. Dynamic instability of microtubule growth. Nature. 1984; 312:237-242. [PubMed: 6504138]

6. Kirschner M, Mitchison T. Beyond self-assembly: from microtubules to morphogenesis. Cell. 1986; 45:329-342. [PubMed: 3516413] 